\title{
Enhanced Manta-Ray Foraging Optimization Algorithm based DCNN for Lane Detection
}

\author{
Snehal S. Shinde \\ JSPM NTC \\ Snehalshindeshinde4@gmail.com
}

\begin{abstract}
In recent days, a developed driver support system has been introduced to enhance driving, which is considered as the renowned one. In the Advanced Driver assistive systems, Lane departure detection plays an important role and it enhances the vehicle's active safe driving. Nowadays, a decent lane detection model that is on the basis of the computer vision methods, is introduced in many studies. From a stream of videos, the lane boundaries and its radius of curvatures and lane direction is detected. This video footage is recorded from a camera mounted on the top of a vehicle. Here, the lane detection model is proposed, which concentrates more on driving assistance. In this paper, a deep learning scheme is used for a lane detection model. Here, the adopted strategies possess two main components namely lane detection and image transformation. At first, the multiple lane images are obtained by the adopted technique and it transforms the image, and it aids in classifying the training. The Deep Convolution Neural Network (DCNN) classifier is considered to detect the lane from the bird's eye view image. An Enhanced Manta-Ray Foraging Optimization Algorithm (EMRFOA) is proposed to aid the DCNN classifier with the optimal weights in this paper. Finally, the developed model is analyzed with the conventional models in that the performance of the adopted model is higher than the conventional models.
\end{abstract}

Keywords: lane detection, deep learning, DCNN, bird's eye view image, optimal weights

\begin{tabular}{ll} 
Nomenclature & \\
\hline Abbreviations & Descriptions \\
\hline DCNN & Deep Convolution Neural Network \\
EMRFOA & Enhanced Manta-Ray Foraging Optimization Algorithm \\
ROI & Region of Interest \\
OBL & Opposition Based Learning \\
DQLL & Deep Q-Learning Localizer \\
FR-IQA & Full-Reference Image Quality Assessment \\
\hline
\end{tabular}

\section{Introduction}

Nowadays, Advanced Driver Assistance Systems become more and more popular. Avoiding accidents as well as guiding the vehicles travel along suitable lanes are two important tasks of those auxiliary systems. Numerous technical essentials are developed to understand these two objectives, namely lane detection and road detection. Advanced development in intelligent transportation, environment perception, as an important task for independent driving has to turn out to be one of the important research trends. For environmental perception, lane detection is considered a significant segment. From the past decades, numerous efforts were performed. Nevertheless, it is considered as confronts to model a robust detector in limitless circumstances. Due to there are numerous variables are presented namely rain, fog, illumination deviation, and partial occlusion. In the ultimate outcomes, they might possess numerous effects [1].

Towards autonomous vehicles, lane detection is a helpful method that can aid cars to alter their location and drive beside the road. For all drivers, recognizing the lanes on the roads is a general task to assure the vehicles within the lane restrict when driving and to minimize the collision chance with other vehicles because of the crossing lanes. Additionally, it is considered an important task for independent vehicles. In order to attain lane detection, it has been shown it is probable to exploit the computer vision model. For independent vehicles, lane detection is exploited to plan for driving routes as well as present safety assurance. Lane detection is a significant task of an intelligent vehicle driving aiding system [2]. 
Speedy and effectual lane detection in road image is not only obliging to aid the planning of path, vehicle counterbalance caution, and traffic flow analysis, other than it can offer a reference for specific navigation. Generally, lanes are not straight lines in practical applications [9] [10]. For instance, lanes are curved at corners; therefore the overview ability of the conventional technique to find the straight lines using the Hough transform is not powerful [3]. Additionally, the majority of the lane detection techniques can merely distinguish fixed-number lanes that were not obtainable towards alternative lane detection.

In heuristic-based lane detection techniques, the preprocessing step plays a significant role. Numerous filters are exploited to eradicate the contaminated noise such as median, mean, Gaussian, and FIR filters. To cope up with the enlightened deviation; the usual solutions use threshold segmentation techniques, such as PLSF, Otsu, and so on. Generally, the ROI is exploited to minimize outmoded information. Disappearance point-based ROI, fixed-size ROI, and adaptive ROI were extensively explored. For pre-processing, color is information. Generally, color space conversion among RGB and YCbCr or HLS is exploited to improve the lane mark quality [4].

The major contribution of this work is to propose and design a developed DAS model for the reliable recognition of road lanes. At first, in the DAS, the multiple lane images are subjected. Subsequently, image transformation is performed that includes the exploit the cameras in the vehicles to capture the views from the cameras. Additionally, it presents the area at the rear of the car to the driver. By exploiting the DCNN model the lane detection is performed and also the optimal weights are computed using the EMRFO algorithm. By tuning the weights optimally in the classifier the efficient lane detection is performed with the aid of the optimization approach.

\section{Literature Review}

In 2020, Jigang Tang et al [1], worked on lane detection technique such as conventional lane detection techniques and associated deep learning techniques. Then, the conventional lane detection techniques were grouped into two steps as two-step and one-step models. The lane detection techniques were introduced from network architectures for lane matching, and few optimization strategies; associated loss functions.

In 2020, Zhiyuan Zhao et al [2], introduced deep reinforcement learning into superficial lane detection techniques for precise lane localization and detection. This technique comprises two components such as landmark point localizer as well as bounding box detector. Subsequently, an enforcement-based DQLLprecisely was proposed to localize the lanes as a group of landmarks to attain a superior symbol of curved lanes.

In 2020, K. Dinakaran et al [3] developed a computer vision-based technique for lane detection model that precisely detects the lanes and curves on the road and presents a prior warning to the drivers. Hence, numerous accidents can be reduced because of the excess speed of vehicles on the curved areas of the road.

In 2020, Raja Muthalagu et al [4], presented a perception technique that was based simply on vision or camera data. A powerful end-to-end lane detection technique was demonstrated by exploiting the upto-date computer vision models for self-driving cars. Initially, a minimalistic technique was presented on the basis of the edge detection and polynomial regression that was the baseline technique to detect merely the straight lane lines. Subsequently, an enhanced lane detection model was proposed on the basis of perspective transformations and histogram analysis.

In 2020, Ge Zhang et al [5], proposed an excellence-guided lane detection method by modeling complicated traffic situations. Here, an uneven number of lanes can be adequately handled. Then, image ROI was captured that only comprises lane information by exploiting the image quality scores and leveraging the prior knowledge. After, an end-to-end two-stage Convolutional Neural Network architecture was designed for lane detection, whereas for lane matching, a binary lane mask was used.

In 2019, Jingyi Liu [6], developed a lane detection model for autonomous vehicles by learning a full reference quality-aware discriminative gradient deep technique. Then, the FR-IQA technique was used to recognize more discriminative gradient cues, and geometric attributes were used concurrently. Then, a recurring neural layer was modeled to indicate the spatial distribution of recognition.

\section{Proposed Lane Detection Model}

In this section, the new lane detection model with the proposed optimization algorithm based on DCNN is presented. Figure 1 demonstrates the architecture model of the detection process. 


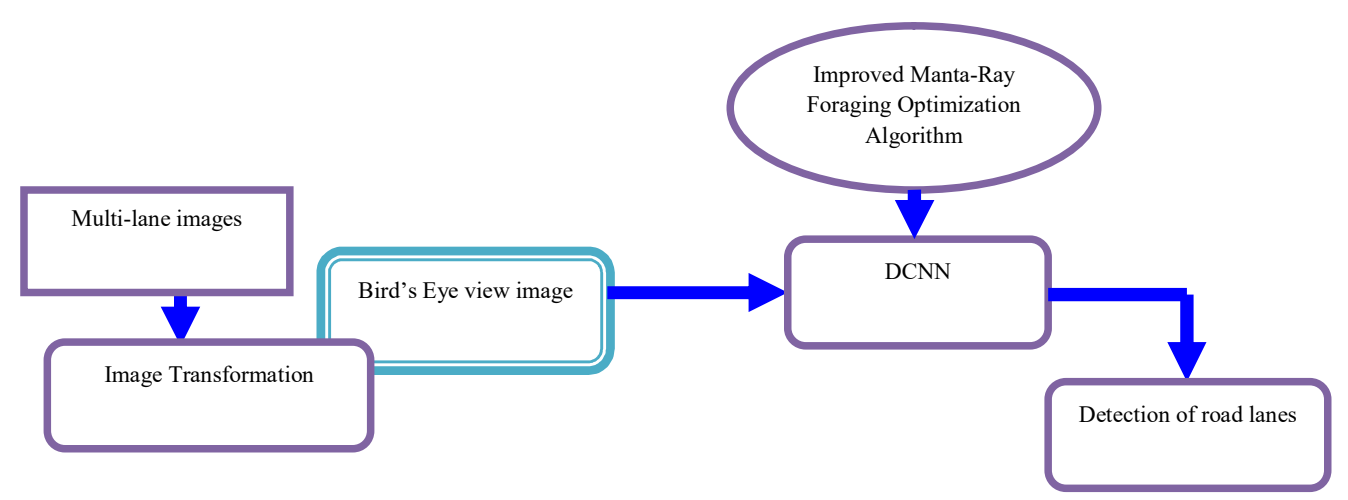

Fig.1 Architecture model of the adopted lane detection

From the multiple road lane images, the adopted model tries to detect the lane, which helps in driving. Using the image classification and transformation, the model carries out the lane detection is performed. By exploiting the camera, multiple images are taken and that is subjected as the training input for the image transformation. Here, the inverse mapping model is considered by the image transformation model, which is exploited to transfer the various lane images to the bird's eye view image. Subsequent to this, this transferred image is subjected to the DCNN classifier; here the developed optimization algorithm is used to train for the effectual lane detection.

\subsection{Image Transformation}

The first step, for the input image sample, in the developed model is to obviously identify the bird's eye view image that is the multi-lane image. As discussed in [7] for the image transformation, this paper considers the Inverse perspective mapping, and the transformation process is stated as below:

\section{a. Body-scaled obstacle avoidance}

The bird's eye view image is produced using the many lanes images with the aid of diverse transformation models in the IPM model. The motivation for the transformation of the image is altering the various lane images into the image which appropriately fits the camera frame. The transformation process is stated as follows:

Initially, by exploiting the method called, body scale obstacle evasion the several obstacles of the many road lane images are alienated.

Consider the spectator is traveling in the straight plane by means of a stable of translation $\mathrm{c}$, and the motion vector proportional to the camera frame is stated as $\frac{\mathrm{dp}}{\mathrm{dt}}=-\mathrm{c}$. The motion filed is a constant that is exploited and stated as $\mathrm{M}(\mathrm{x}, \mathrm{y}, \mathrm{z}) \mathrm{using}$ the eq. (1),

$$
\mathrm{M}^{\prime}(\varphi(p))=\frac{d \varphi(p)}{d t}=-J \varphi(p) \cdot c
$$

whereas, $\varphi(\mathrm{p})$ represents the image perspective projection, $-\mathrm{c}$ represents the motion vector associated with the camera frame, and J represents the Jacobian matrix measured for the image perspective projection. After carrying out the initial step, the IPM is used to the eq. (1), to find out the altered image, and it is indicated as,

$$
\mathrm{M}^{\mathrm{T}}=-\mathrm{J} \varphi(\mathrm{p}) \cdot \mathrm{c}=-\mathrm{J} \varphi^{\mathrm{T}}(\mathrm{p}) \cdot \mathrm{c}
$$

Additionally, to the bird's eye view image $\mathrm{M}^{\mathrm{T}}$ the abundant lane images of transformation $\mathrm{M}$ based on the transformation parameter $\mathrm{T}$, and it is indicated as,

$$
\mathrm{M}^{\mathrm{T}^{\mathrm{T}}}=-\mathrm{T}\left(\begin{array}{ccc}
-\frac{1}{\mathrm{p}^{3}} & 0 & -\frac{\mathrm{p}_{1}}{\frac{2}{3}} \\
0 & -\frac{1}{\mathrm{p}^{3}} & \frac{\mathrm{p}_{1}}{\mathrm{p}^{\frac{2}{3}}}
\end{array}\right)\left(\begin{array}{l}
\mathrm{c}_{1} \\
c_{2} \\
\mathrm{c}_{3}
\end{array}\right)
$$

The emotion plane comprises the image emotion, and consequently, the changed image can be stated as

$$
\mathrm{M}^{\mathrm{T}}=\frac{\mathrm{T}}{\mathrm{p}_{3}}\left(\begin{array}{l}
\mathrm{c}_{1} \\
\mathrm{c}_{2}
\end{array}\right)
$$

Finally, the abundant lane images are altered into the bird's eye view image and are indicated as, 


$$
\left\|\mathrm{M}^{\prime} \mathrm{T}\right\|=\left|\frac{\mathrm{T}}{\mathrm{T}-\mathrm{elev}}\right| \cdot\|\mathrm{c}\|
$$

whereas, elev indicates the elevation point, and the elevation point value is stated as $\mathrm{T}+\mathrm{p}_{3}$.

\subsection{Lane detection using adopted model}

From the sample image I, the bird's eye view image B is detected; the image is directly subjected to the DCNN classifier. Moreover, the optimal DCNN classifier weights are chosen exploiting the new method, and therefore, the classifier is called EMRFOA based DCNN [11]. By presenting both the input and its equivalent ground information the detection process is performed.

\section{a. Adopted model architecture}

Figure 2 demonstrates the proposed lane detection architecture with EMRFOA based DCNN model. The DCNN [8] consists of several layers, namely Fully Connected (FC) layers, convolutional layers (Conv layers), pooling (POOL) layers.

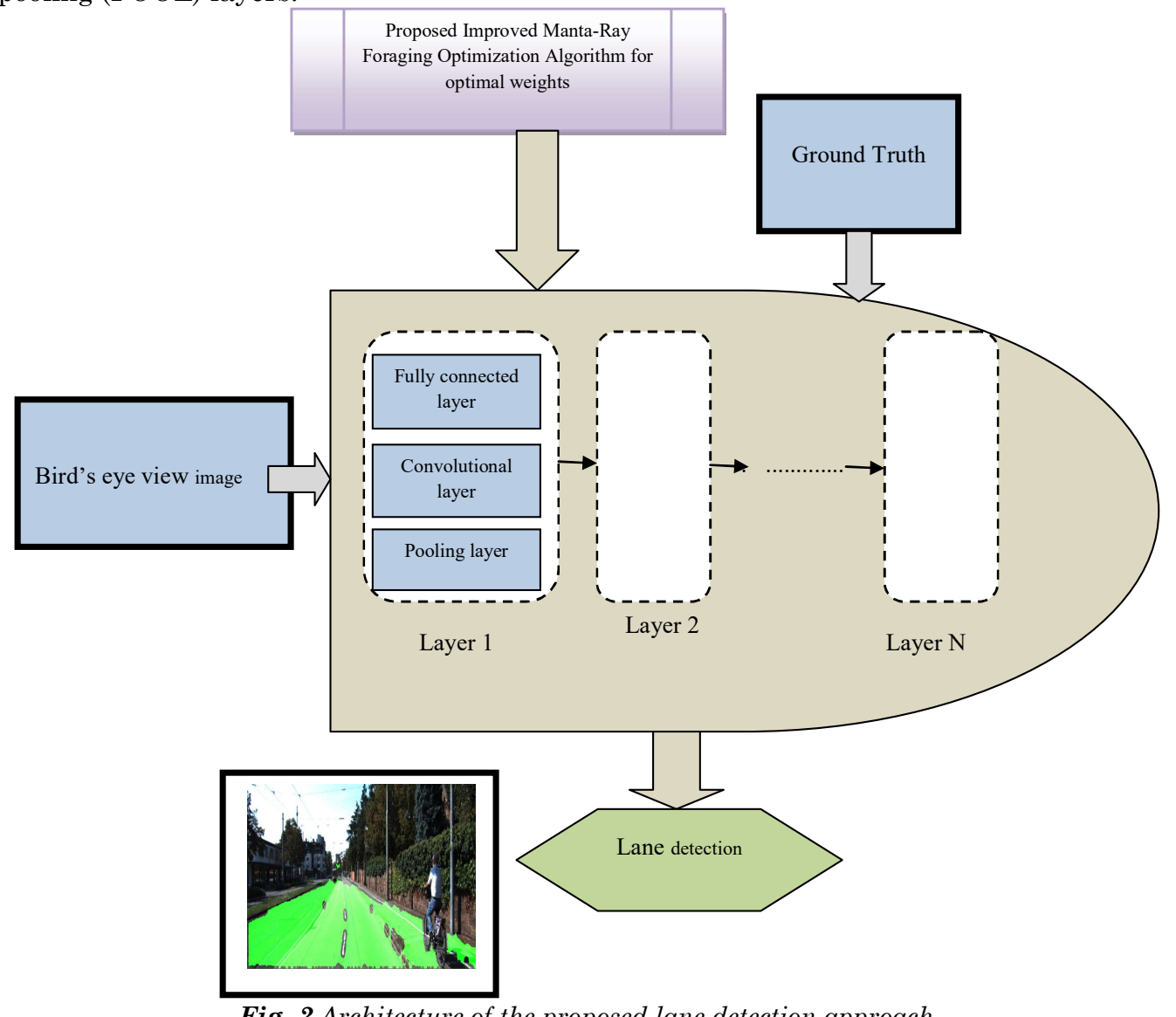

The DCNN attains the bird's eye view image for the training and it is directly passed to the conv layer. By exploiting the POOL layer, the subsampling is performed as well as the output is attained from the FC layer.

Conv layers: In the DCNN classifier, the bird's eye image is subjected as the input of the initial conv layer. This classifier comprises several conv layers and using the weight parameters they are unified. By exploiting the transformed image and the weight, the feature map is created, which is exploited for the training. Subsequently, in the conv layer, the details are presented to the other, which are subjected to the activation function. Using eq. (6), the DCNN classifier Conv layer is demonstrated,

$$
\mathrm{K}=\left\{\mathrm{K}_{1}, \mathrm{~K}_{2}, \ldots, \mathrm{K}_{\mathrm{V}}, \ldots, \mathrm{K}_{\mathrm{m}}\right\}
$$

whereas, the DCNN mindicates a total number of the conv layer, $\mathrm{K}_{\mathrm{v}}$ denotes $\mathrm{v}^{\text {th }}$ conv layer. The conv layer output is calculated as below, 


$$
\left(Y_{d}^{v}\right)_{r, s}=\left(B_{d}^{v}\right)_{r, s}+\sum_{e=1}^{J_{1}^{e-1}} \sum_{p=-n_{1}^{j}}^{n_{1}^{j}} \sum_{q=-n_{2}^{j}}^{n_{2}^{j}}\left(G_{d, e}^{v}\right)_{p, q} *\left(Y_{e}^{v-1}\right)_{r+p, s+q}
$$

whereas, * indicates the operator in the conv layer, $\left(\mathrm{B}_{\mathrm{d}}^{\mathrm{v}}\right)_{\mathrm{r}, \mathrm{s}}$ states the bias $\left(\mathrm{Y}_{\mathrm{e}}^{\mathrm{v}-1}\right)_{\mathrm{r}+\mathrm{p}, \mathrm{s}+\mathrm{q}}$ indicates the conv layer's input by using the feature map, $\left(G_{d, e}^{\mathrm{v}}\right)_{p, q}$ states the weight of the conv layer, and $\left(\mathrm{Y}_{\mathrm{d}}^{\mathrm{v}}\right)_{\mathrm{r}, \mathrm{s}}$ states the output from the $\mathrm{v}^{\text {th }}$ conv layers or fixed feature map centered as $(\mathrm{r}, \mathrm{s})$.

ReLU and POOL layer: ReLU is exploited to offer the adaptable output of the conv layer exploiting the activation function, and it assures the efficiency, and it is stated as,

$$
\mathrm{Y}_{\mathrm{d}}^{\mathrm{v}}=\operatorname{fun}\left(\mathrm{Y}_{\mathrm{d}}^{\mathrm{v}-1}\right)
$$

whereas, fun( $)$ states the activation function stated for the $\mathrm{v}^{\text {th }}$ layer. Subsequently, to the POOL layer, the ReLU layer's output is subjected as the input. By the POOL layer, the local regions of the input are processed, without exploiting the weights and biases.

FC layers: Subsequently, the POOL layer results are fed to the FC layer's input. The classification is carried out by exploiting the high-level reasoning in the FC layer, and the result is formulated as,

$$
\mathrm{Z}_{\mathrm{d}}^{\mathrm{v}}=\mathrm{T}\left(\mathrm{b}_{\mathrm{d}}^{\mathrm{v}}\right) \text { with } \mathrm{b}_{\mathrm{d}}^{\mathrm{j}}=\sum_{\mathrm{e}=1}^{\mathrm{J}_{1}^{\mathrm{e}-1}} \sum_{\mathrm{r}=1}^{\mathrm{J}_{2}^{\mathrm{e}-1}} \sum_{\mathrm{s}=1}^{\mathrm{J}_{3}^{\mathrm{e}-1}}\left(\mathrm{U}_{\mathrm{d}, \mathrm{e}, \mathrm{r}, \mathrm{s}}^{\mathrm{v}}\right) \cdot\left(\mathrm{Y}_{\mathrm{d}}^{\mathrm{v}-1}\right)_{\mathrm{r}, \mathrm{s}}
$$

whereas, $\mathrm{U}_{\mathrm{d}, \mathrm{e}, \mathrm{r}, \mathrm{s}}^{\mathrm{v}}$ indicates the weight interlinking $(\mathrm{r}, \mathrm{s})$ of the $\mathrm{e}^{\text {th }}$ feature map of layer $\mathrm{v}-1$ and $\mathrm{d}^{\text {th }}$ unit in the layer $\mathrm{j}$. The output is produced by exploiting the weights of the three layers with the aid of the proposed optimization technique. The optimization technique is exploited to estimate the optimal weights of the DCNN classifier that is indicated as, $D \in\{B, G, U\}$.

\section{Proposed Optimization Method to find the Optimal Weights}

In this paper, for the DCNN classifier, the optimization model has been proposed to calculate the optimal weights. The proposed EMRFOA optimization model [9] is a novel bio-inspired optimization technique that presents a discontinuous optimization technique. MRFO approach is a novel bio-inspired optimization algorithm which gives an alternating optimization model to focus on here and now engineering problems. This technique is inspired by manta rays' intelligent performances. Here, 3 different foraging manta rays schemes, containing chain foraging, cyclone foraging, and somersault foraging is assuemed, to provide an effective optimization model to find solutions for various optimization issues.

In every iteration, the location is updated by the optimal position prey with more planktons.

$$
x_{i}^{d}(t+1)=\left\{\begin{array}{l}
x_{i}^{d}(t)+r \times\left(\left(x_{d}^{\text {best }}(t)-x_{i}^{d}(t)\right)\right) \\
+\alpha \times x_{\text {best }}^{d}(t)-x_{i}^{d}(t), i=1 \\
x_{i}^{d}(t)+r\left(\left(x_{i-1}^{d}(t)-x_{i}^{d}(t)\right)\right) \\
\alpha \times x_{\text {best }}^{d}(t)-x_{i}^{d}(t), i=2, \ldots ., N
\end{array}\right.
$$

whereas $\alpha$ indicates the weight coefficient, $r$ indicates the random number among 0 and $1, \mathrm{x}_{\text {best }}^{\mathrm{d}}(\mathrm{t})$ indicates an individual plankton density, $\mathrm{x}_{\mathrm{i}}^{\mathrm{d}}(\mathrm{t})$ indicates the location of $i^{\text {th }}$ and $\mathrm{x}_{\mathrm{i}-1}^{\mathrm{d}}(\mathrm{t})$ the (i-1)th representative at the time $t$ in $d$ th dimension and $\alpha$ are attained as below: 


$$
\begin{aligned}
& \alpha=2 r \times|\log (r)|^{1 / 2} \\
& x_{i}^{d}(t+1)=\left\{\begin{array}{l}
x_{i}^{d}(t)+r \times\left(\left(x_{d}^{\text {best }}(t)-x_{i}^{d}(t)\right)\right) \\
+\beta \times x_{\text {best }}^{d}(t)-x_{i}^{d}(t), i=1 \\
x_{i}^{d}(t)+r\left(\left(x_{i-1}^{d}(t)-x_{i}^{d}(t)\right)\right) \\
\beta \times x_{\text {best }}^{d}(t)-x_{i}^{d}(t), i=2, \ldots, N
\end{array}\right. \\
& \beta=2 \exp \left(r_{1} \times\left(\frac{T-t+1}{T}\right)\right) \times \sin \left(2 \pi r_{i}\right)
\end{aligned}
$$

whereas $\beta$ indicates the weight coefficient, $T$ indicates the maximum amount of iteration, and $r_{1}$ indicates the random number among optimal solutions at random. The storm performance is attained to improve the exploration of the technique to attain the most appropriate random position solution.

$$
\mathrm{x}_{\text {rand }}^{\mathrm{d}}=\mathrm{L}^{\mathrm{d}}+\mathrm{r} \times\left(\mathrm{u}^{\mathrm{d}}-\mathrm{l}^{\mathrm{d}}\right)
$$

Hence, these positions were to assumed attain the optimum location.

$$
x_{i}^{d}(t+1)=x_{i}^{d}+S \times\left(r_{2} \times x_{\text {best }}^{d}-r_{3} \times x_{i}^{d}\right) i=1,2, \ldots ., N
$$

whereas

$$
r_{2}
$$

$\mathrm{r}_{3}$ indicates two random amounts between 0 and 1and $\mathrm{S}$ indicates the somersault bait and amounts to 2 . Two enhancements are performed in this . method. Initially, the method is modified using a well-known technique, named OBL method. A divergence from most important solution than the optimal solution is seen sometimes. The OBL method was used to obtain further effectual results in the technique individual as below: $\mathrm{x}_{\mathrm{i}}^{-\mathrm{d}}=\mathrm{x}_{\mathrm{i}}^{\max }+\mathrm{x}_{\mathrm{i}}^{\min }-\mathrm{x}_{\mathrm{i}}^{\mathrm{d}}$

whereas, $x_{i}^{-d}$ indicates the contrary location for the $x_{i}^{d}$, and $x_{i}^{\max }$ and $x_{i}^{\min }$ indicates the maximum and the minimum constraints. Then, $x_{i}^{-d}$ is put in place of $x_{i}^{d}$, if $x_{i}^{-d}$ has proper attainments than $x_{i}^{d}$. Using the self-adaptive method is the next enhancement.

$$
\mathrm{x}_{\mathrm{i}}^{\mathrm{d}}(\mathrm{t})=10 \times \mathrm{N}
$$

Subsequent to, the updated individual size for the afterward iteration is
astained
using
$\mathrm{x}_{\mathrm{i}}^{-\mathrm{d}+}=\operatorname{round}\left(\mathrm{x}_{\mathrm{i}}^{\mathrm{d}} \times(1+\delta)\right)$

whereas, $\delta$ indicates a random uniform distribution between in the range $[-0.5,0.5]$, that indicates an adjustable variable

\section{Result and Discussion}

In this section, the experimentation outcomes of the proposed lane detection model were modeled. The outcomes were examined by representing the images from the database. It was especially modeled for the lane detection techniques and it possesses a complete of 289 training images and 290 testing images. There are three types of road images in the database namely urban marked, urban multiple marked lanes, and urban unmarked. Here, the proposed method is compared with the conventional models such as Convolutional Neural Network (CNN), Neural Network (NN), and Crow Search Algorithm (CSA).

Table 1 summarizes the analysis of the developed model-based DCNN classifier for several epoch values over training data. The performance analysis is performed by varying both the training percentage and $\mathrm{k}$-fold the values of the image samples from the database. The assessment of the proposed model is performed over the three estimate measures for both the analysis. 
Table 2 summarizes the performance analysis of the proposed model by varying the training percentage of the data samples from the database. Here, the proposed method is $15 \%$ better than the CNN, $17 \%$ better than the NN, $18 \%$ better than the CSA for detection accuracy in 0.5 training percentage.

Table 1 Comparative analysis of the proposed technique

\begin{tabular}{|c|c|c|c|c|c|}
\hline $\begin{array}{c}\text { Training } \\
\text { Percentage }\end{array}$ & 0.5 & 0.6 & 0.7 & 0.8 & 0.9 \\
\hline Epoch & \multicolumn{5}{|c|}{ Detection Accuracy } \\
\hline 20 & 0.8504 & 0.8565 & 0.8651 & 0.8778 & 0.8784 \\
\hline 30 & 0.8386 & 0.8734 & 0.8744 & 0.8788 & 0.8803 \\
\hline 40 & 0.8584 & 0.8787 & 0.8818 & 0.8835 & 0.8858 \\
\hline \multirow[t]{2}{*}{50} & 0.854 & 0.8755 & 0.8804 & 0.8838 & 0.8868 \\
\hline & \multicolumn{5}{|c|}{ Sensitivity } \\
\hline 20 & 0.8337 & 0.8431 & 0.8616 & 0.8743 & 0.8813 \\
\hline 30 & 0.8338 & 0.8653 & 0.8663 & 0.8673 & 0.8887 \\
\hline 40 & 0.8558 & 0.8736 & 0.8768 & 0.8816 & 0.8843 \\
\hline \multirow[t]{2}{*}{50} & 0.8688 & 0.8753 & 0.8835 & 0.8861 & 0.8865 \\
\hline & \multicolumn{5}{|c|}{ Specificity } \\
\hline 20 & 0.8706 & 0.8713 & 0.8313 & 0.8336 & 0.8336 \\
\hline 30 & 0.8065 & 0.8088 & 0.8307 & 0.8308 & 0.8543 \\
\hline 40 & 0.8003 & 0.8358 & 0.8378 & 0.8468 & 0.8567 \\
\hline 50 & 0.8788 & 0.8865 & 0.8186 & 0.8383 & 0.8334 \\
\hline
\end{tabular}

Table 2 Comparative analysis of the proposed technique regarding the percentage variation

\begin{tabular}{|c|c|c|c|c|c|}
\hline $\begin{array}{c}\text { Training } \\
\text { Percentage } \\
\end{array}$ & 0.5 & 0.6 & 0.7 & 0.8 & 0.9 \\
\hline Methods & \multicolumn{5}{|c|}{ Detection Accuracy } \\
\hline CNN & 0.8824 & 0.8916 & 0.9133 & 0.9222 & 0.928 \\
\hline NN & 0.747 & 0.749 & 0.787 & 0.795 & 0.801 \\
\hline CSA & 0.943 & 0.9475 & 0.9725 & 0.98 & 0.9858 \\
\hline Proposed model & 0.963 & 0.9688 & 0.971 & 0.985 & 0.9951 \\
\hline & \multicolumn{5}{|c|}{ Sensitivity } \\
\hline CNN & 0.9351 & 0.9547 & 0.9663 & 0.9789 & 0.9871 \\
\hline NN & 0.8858 & 0.9092 & 0.9342 & 0.9383 & 0.96 \\
\hline CSA & 0.9454 & 0.9726 & 0.9803 & 0.9839 & 0.9852 \\
\hline Proposed model & 0.9542 & 0.9606 & 0.9733 & 0.9915 & 0.9925 \\
\hline & \multicolumn{5}{|c|}{ Specificity } \\
\hline CNN & 0.745 & 0.745 & 0.7901 & 0.8045 & 0.9194 \\
\hline NN & 0.7655 & 0.784 & 0.7937 & 0.8012 & 0.8269 \\
\hline CSA & 0.895 & 0.91 & 0.9362 & 0.9742 & 0.9851 \\
\hline Proposed model & 0.8783 & 0.895 & 0.92 & 0.97 & 0.995 \\
\hline
\end{tabular}

\section{Conclusion}

A lane detection model was proposed to aid the driver assistance system in this work. To the image transformation, the multiple lane images which were captured using the camera were subjected as the training input. Here, the inverse mapping model was exploited for the image transformation. After the image transformation, in order to detect the lane, the bird's eye view image was subjected to the DCNN classifier. To tune the optimal weights the proposed method was exploited, which trains the DCNN classifier on the basis of the lane detection, which was calculated. Finally, the experimentation of the developed model using the proposed optimization method-based DCNN classifier was performed by contemplating the multiple lane images from the standard database. Finally, the performance of the proposed method was performed by exploiting the various measures, like specificity, sensitivity, and detection accurateness. The proposed model possesses a maximum detection rate, and as well it works precisely and robustly in heavy traffic

\section{References}

[1] Jigang TangSongbin LiPeng Liu,"A review of lane detection methods based on deep learning", Pattern Recognition, 15 September 2020.

[2] Zhiyuan ZhaoQi WangXuelong Li,"Deep reinforcement learning based lane detection and localization", Neurocomputing, 9 July 2020. 
[3] K. DinakaranA. Stephen SagayarajGokul Chandrasekaran,"Advanced lane detection technique for structural highway based on computer vision algorithm", Materials Today: ProceedingsAvailable online, 24 October 2020.

[4] Raja MuthalaguAnudeepsekhar BolimeraV. Kalaichelvi,"Lane detection technique based on perspective transformation and histogram analysis for self-driving cars", Computers \& Electrical Engineering, 18 May 2020.

[5] Ge ZhangChaokun YanJianlin Wang,"Quality-guided lane detection by deeply modeling sophisticated traffic context", Signal Processing: Image Communication,19 February 2020.

[6] Jingyi Liu,"Learning full-reference quality-guided discriminative gradient cues for lane detection based on neural networks",Journal of Visual Communication and Image Representation,9 October 2019.

[7] Mallot, H.A., Bülthoff, H.H., Little, J.J and Bohrer, S. (1991). Inverse perspective mapping simplifies optical flow computation and obstacle detection, Biological cybernetics, 64(3): 177-185.

[8] Rakhlin, A., Shvets, A., Iglovikov, V and Kalinin, A.A. "Deep Convolutional Neural Networks for Breast Cancer Histology Image Analysis, International Conference Image Analysis and Recognition”, ICIAR, Image Analysis and Recognition, pp. 737-744, 2018.

[9] Jiaying FengXiaoguang LuoSomayeh Pouramini,"Minimization of energy consumption by building shape optimization using an improved Manta-Ray Foraging Optimization algorithm", Energy Reports, 15 February 2021.

[10] K.Srinivas,"Prediction of E-Learning Efficiency by Deep Learning in EKhool Online Portal Networks", Multimedia Research, vol. 3, no. 4, October 2020

[11] Arvind Madhukar Jagtap,"Developing Deep Neural Network for Learner Performance Prediction in EKhool Online Learning Platform",Multimedia Research, vol. 3, no. 4, October 2020.

[12] G.Gokulkumari,"Classification of Brain Tumor using Manta Ray Foraging Optimization-based Deep CNN Classifier",Multimedia Research, vol. 3, no 4, October 2020. 